\title{
Optimization and Long-Term Stability of Micro Flow Sensors for Smart VP Shunts
}

\author{
Gergő Édes, Eniko T. Enikov, Jesse Skoch and Rein Anton
}

\begin{abstract}
This paper reports on a systematic study of the flow sensitivity and resolution of micro-flow sensors intended for use in implantable ventricular-peritoneal shunts. The flow sensors utilize ferromagnetic flaps (transducers) whose deflection is detected by ultra-sensitive MTJ sensors $(20 \mathrm{mV} / \mathrm{V} / \mathrm{Oe})$. A working range of $0-40 \mathrm{ml} / \mathrm{h}$ was demonstrated at a maximum uncertainty of $4 \% \mathrm{RMS}$ and a resolution of $0.4 \mathrm{ml} / \mathrm{h}$. Earlier studies on this sensor unveiled significant low-frequency noise (drift) limiting the sensitivity to $1.4 \mathrm{ml} / \mathrm{hr}$. The present study identifies thermal noise as the main source of low-frequency drift. Using thermal compensation it was found that the drift can be reduced below $2 \mathrm{ml}$ per 24-hr. Combining an array of four transducers operating in series, it has been demonstrated that a sensitivity can be increased $\mathbf{1 0 . 9}$ fold. Furthermore, the report examines the long-term structural stability of the sensors and produces a corrosion report suggesting a lifespan of 15 to 55 years.
\end{abstract}

Index Terms-VP shunt; Flow sensor; Thermal noise; MEMS; MTJ sensor

\section{INTRODUCTION}

$\mathbf{M}$ AGNETIC tunneling junction (MTJ) sensors are finding ever more diverse applications. One such area is the detection of slow-moving fluids in medical catheters. Drainage of cerebral spinal fluid is important in the treatment of hydrocephalus, which has an incidence rate of 5.5 per 100,000 [1]. A common failure mode of implanted cerebrospinal fluid shunts is occlusion or fouling of the catheter which occurs in $40 \%$ of the implanted shunts during the first year, in $50 \%$ by the end of the first two years and as high as $80 \%$ during the lifetime of the implant [2], [3], [4]. Today around $10 \%$ of deaths related to hydrocephalus are caused by shunt failure, despite significant advances in shunt design. [5]. The height failure rate leads to over 27,000 surgeries every year that are aimed to repair or diagnose a failing shunt [6].

The need for continuous monitoring of the shunt flow has long been recognized, leading to the first published account of a telemetric, in-line, implanted device due to Atkinson et al. in 1967. [7]. Instead of flow measurements, the device measured changes in intra-cranial pressure (ICP). Efforts in the early 1980s were aimed at producing a batteryless prototype [8], however without demonstration of a functioning unit. More recent efforts showcased prototypes relying on calorimetric principles to measure flow rate using external power sources

ET. Enikov and G. Edes are with the Department of Aerospace and Mechanical Engineering, University of Arizona, Tucson, AZ, 85721 USA e-mail: enikov@engr.arizona.edu

J Skoch and R. Anton are with the Department of Surgery, University of Arizona, Tucson, AZ, 85721

Manuscript received xx, 2014; revised xxx, 2014. and readers. Bork et al. [9] demonstrated a sensor range of 2$40 \mathrm{ml} / \mathrm{h}$ with $\mathrm{a} \pm 10 \%$ uncertainty, Qin et al.[10] achieved a working range of $0-52 \mathrm{ml} / \mathrm{h}$ with a $6.3 \% \mathrm{RMS}$ error collecting samples every $30 \mathrm{~s}$, Hudson et al. [11], used impedance measurements of transiently heated Cerebrospinal Fluid (CSF) to demonstrate sensitivity of $2.9 \mathrm{ml} / \mathrm{hr}$ and resolution of 3 $\mathrm{ml} / \mathrm{h}$. Flow sensing based on hydraulic pressure difference was demonstrated my Raj et al. [12] using an external spectrometer to measure passive subcuteneous components with a claimed sensitivity of $0.6 \mathrm{ml} / \mathrm{h}$.

This paper presents the development of a self-powered, self-contained implantable flow sensor using an alternative detection method based on magneto-tunneling junction (MTJ) allowing for high frequency continuous data collection. Details of the operation of the sensor were presented in an earlier publication by the authors, [13]. Briefly, the flow is detected by measuring the deflection of a ferromagnetic transducer under the action of the drag force. Initial studies of the response of the sensor showed a significant low-frequency drift with unknown origin. In this work we aim to address the shortcoming of the previous design and elevate it to a viable implantable device with an uncertainty under $6 \%$ and resolution of over $1 \mathrm{ml} / \mathrm{h}$. The sensor is required to continuously collect flow data over 24 hours without any interaction with an external device, using the built-in battery to operate. After 24 hours wireless Near-Field Communication (NFC) data transmission can be used to transfer the data to a $\mathrm{PC}$ for further analysis. Issues with the previously published design will be discussed, showing that the drift was due to uncontrolled thermal fluctuations in the sensor environment. Furthermore, optimizing the geometry of the sensor has led to a 10-fold increase in the signal to noise ratio allowing detection of flows below $1 \mathrm{ml} / \mathrm{hr}$. A 24 -hour volumetric error (integral of the flow rate) of less that $2 \mathrm{ml}$ per 24-hr was also demonstrated.

\section{Materials And Methods}

The transducer is based on detecting Stokes drag through placing a flexible thin flap inside the fluid stream (see Figure 1). The resulting drag causes the flap to bend in the direction of the flow, thus distorting the magnetic field created by a permanent magnet placed under the ferromagnetic nickel transducer. The change in magnetic field is then detected by a magnetic (MTJ) sensor mounted near the movable transducer. The MTJ sensors have directional sensitivity, with the sensitive axis being the direction of the flow. Further details about the working principles of the transducer, the magnetic field 
analysis and the transducer fabrication can be found in Enikov et.al. [13].

A commercially available magnetic field sensor was used to detect the deflection of the transducers. The specific sensor used is the Micromagnetics STJ-240 with 5-20 mv/V/Oe sensitivity. Figure 2 shows the measurement circuit used for detecting resistance change in the MTJ sensor. In addition to the two-stage amplification, the circuit provides low-pass filtering with a cutoff frequency of $3 \mathrm{~Hz}$.

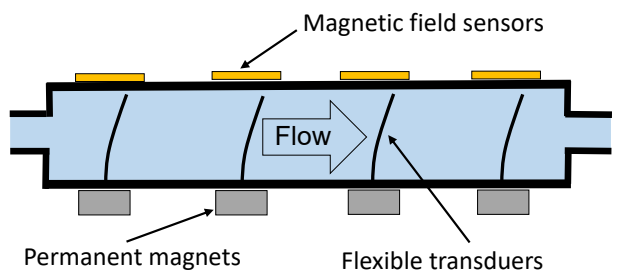

Fig. 1. Flow detection principle.

\section{SENSOR DESIGN}

Under normal physiological conditions, the central nervous system discharges $450-550 \mathrm{ml}$ of CSF per day [14]. This is equivalent of $20 \mathrm{ml} / \mathrm{hr}$. Due to cardiovascular pulsations, the flow is likely pulsatile with an estimated rage of 1$100 \mathrm{ml} / \mathrm{hr}$. Therefore, the desired resolution of the sensor is approximately $1 \mathrm{ml} / \mathrm{hr}$. In a previous study, we have presented a working prototype with a sensitivity of $25 \mathrm{mV} / \mathrm{ml} / \mathrm{h}$, which is easily detectable in the absence of noise. However, lowfrequency drift necessitated a re-design aimed at increasing the signal to noise ratio. The following sections describe these improvements.

\section{A. Low-Frequency Noise Analysis}

One of the greatest limiting factors of the previously published design was the presence of a significant low-frequency signal drift [13]. Possible causes of the drift could be a mismatch between the temperature coefficient of resistance (TCR) of the resistors in the Wheatstone bridge. Another source of thermal noise are the two amplifiers producing a combined gain of 3300. Thus the investigation focused on correlating temperature fluctuations of the environment around the sensor with its output. To this end a laboratory test has been performed where a temperature sensor was attached to the MTJ sensor and recordings were made at $10 \mathrm{~Hz}$ sample rate over a period of 24 hours resulting in 864000 temperature and voltage output data points. During the data collection the flow rate was set to $0 \mathrm{ml} / \mathrm{h}$ in order to isolate the noise from the signal. The data collected is displayed in Figure 3. This figure revealed a strong correlation between the temperature readings and the sensor output. A correlation analysis showed a temperature sensitivity of $3.9 \mathrm{~V} /{ }^{\circ} \mathrm{C}$.

A linear dynamic model was extracted using the temperature signal as its input and the sensor signal as the model output. The model was then used to subtract component due to temperature fluctuations. Since experiments were carried at zero net flow, the compensated signal has zero mean as shown by the red trace in Figure 4. Fourier transforms of the raw (original) signal and the compensated one are shown in Figure 5. As evident from the figure, the highest peak in the spectrum appeared near $0.4 \mathrm{mHz}$, corresponding to a period of about 40 minutes. Most likely this period corresponds to the cycle of the air conditioning system in the lab. Additionally a lower frequency peak near $2.3 \times 10^{-5} \mathrm{~Hz}$ (12 hours) is also noticeable. Its magnitude was also attenuated as a result of the dynamic compensation, resulting in $0.2 \mathrm{~V}$-rms amplitude (flow rate of $10.5 \mathrm{ml} / \mathrm{hr}$-RMS) Over 12-hour period, such drift would constitute a significant volumetric error of about 126 $\mathrm{mL}$, not acceptable for the envisioned application.

Further reduction of the thermal drift requires detailed analysis of each sensor component. These are the three resistors and the magnetic field sensor forming the Wheatstone bridge. Data from the manufacturer indicated that the MTJ sensor has a coefficient ot thermal resistance of $0.0006 \Omega /{ }^{\circ} \mathrm{C}$ and a nominal resistance of $1340 \Omega$. Correspondingly, the anticipated resistance change is $0.8 \Omega$ for every degree Celsius of thermal fluctuation. Assuming that no other component varies with temperature, under the gain of 3300 , the output fluctuation is expected to be $2.456 \mathrm{~V}$ for each degree of temperature change. The measured thermal sensitivity however, was $3.9 \mathrm{~V} /{ }^{\circ} \mathrm{C}$, thus suggesting that other components contribute to the thermal drift. To investigate this, the MTJ sensor was placed in an oven and tested over a range of 26 to $33{ }^{\circ} \mathrm{C}$, while the rest of the circuitry was kept outside at room temperature. The output data and correlation plot of this test are shown in Figure 6. A thermal sensitivity of $2 \mathrm{~V} /{ }^{\circ} \mathrm{C}$ was observed while the value estimated from the MTJ sensor manufacturer's data is 2.456 $\mathrm{V} /{ }^{\circ} \mathrm{C}$. The good agreement between the two values suggest that indeed in addition to the MTJ sensor, other components in the circuit contribute to the overall sensitivity of $3.9 \mathrm{~V} /{ }^{\circ} \mathrm{C}$. Most likely these are the resistors used to complete the bridge. Solutions to reduce the overall temperature sensitivity are presented in the following section by placing four MTJ sensors with a similar TCR in a Wheatstone bridge. This implementation has has reduced the temperature sensitivity from $3.9 \mathrm{~V} /{ }^{\circ} \mathrm{C}$ to $1.1 \mathrm{~V} /{ }^{\circ} \mathrm{C}$

\section{B. Multi-Sensor Array}

The noise amplitude of the temperature compensated signal presented in the previous section corresponds to $\pm 15 \mathrm{ml} / \mathrm{h}$ of uncertainty in the accuracy of the sensor and over $200 \mathrm{ml}$ of volumetric error over 24 hours. This does not meet the sensor specifications stated above, therefore further improvements in sensitivity and noise reduction will be necessary. One solution to increase sensitivity and reduce noise is to introduce multiple sensors that work together. To demonstrate the sensitivity gain, four MTJ magnetic sensors have been implemented in a Wheatstone bridge with four nickel transducers underneath the sensors to help increase the signal to nose ratio. The orientation of the sensors has been optimized for maximum sensitivity when placed in a bridge configuration. The physical placement of the sensors along with their sensitive directions is shown in Figure 7. An additional benefit of using four sensors 


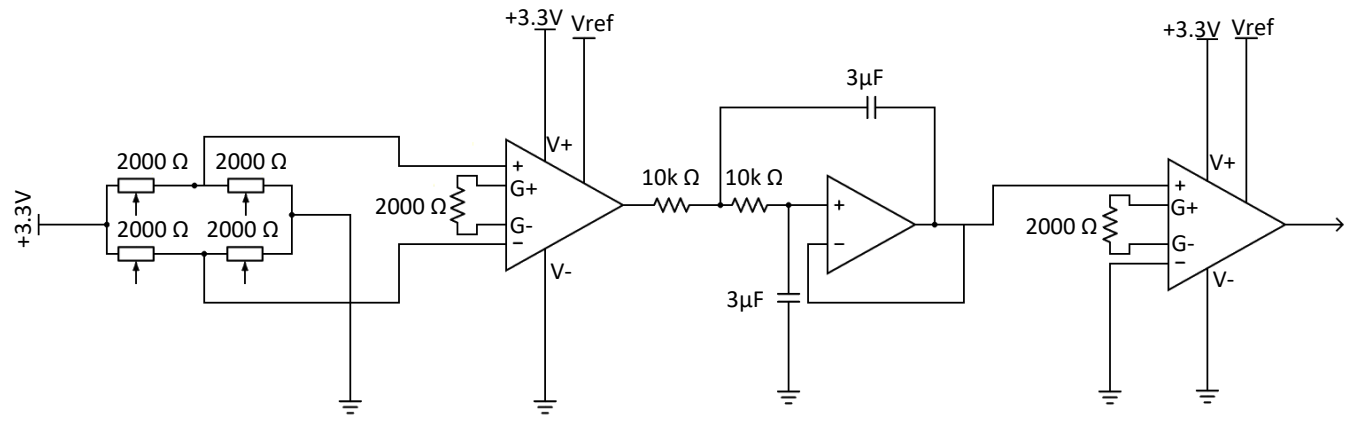

Fig. 2. Sensor signal conditioning circuit.
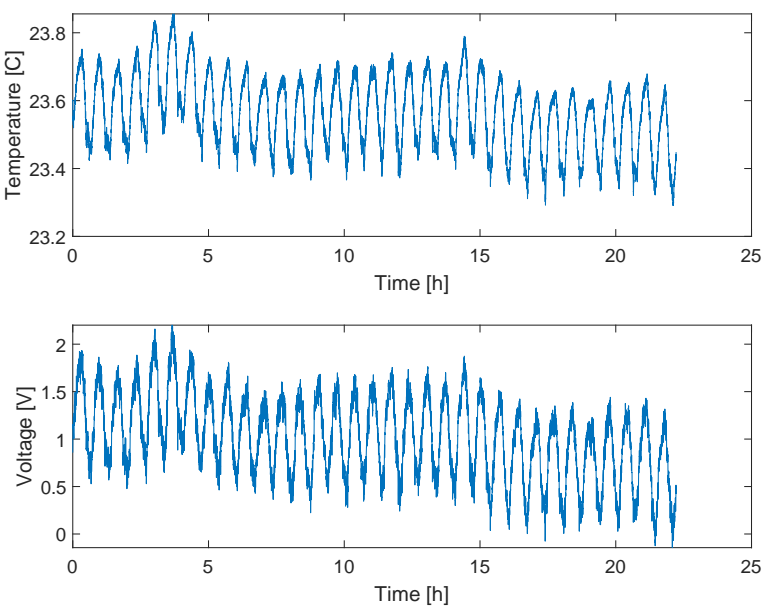

Fig. 3. Data output from temperature and magnetic sensors.

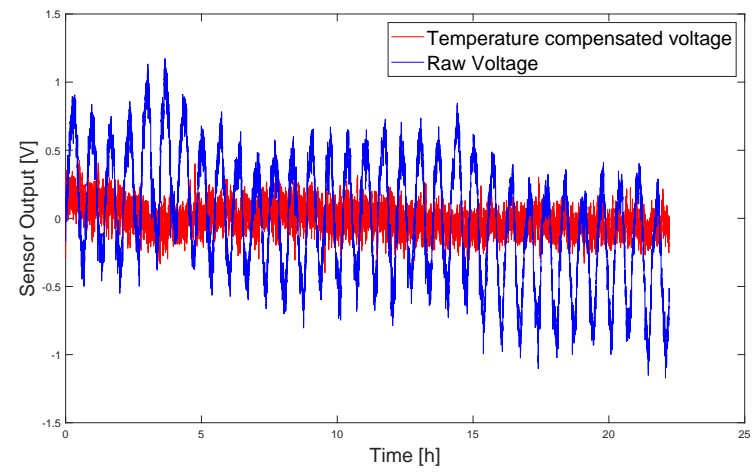

Fig. 4. Raw voltage output vs. temperature compensated output with single MTJ sensor.

in a Wheatstone bridge is the reduced temperature sensitivity of the device due to the similar TCR values of each MTJ sensor.

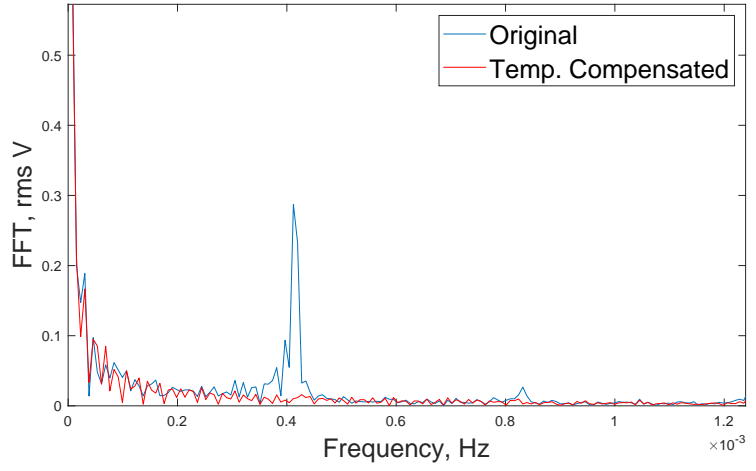

Fig. 5. FFT of original vs. temperature compensated signal.

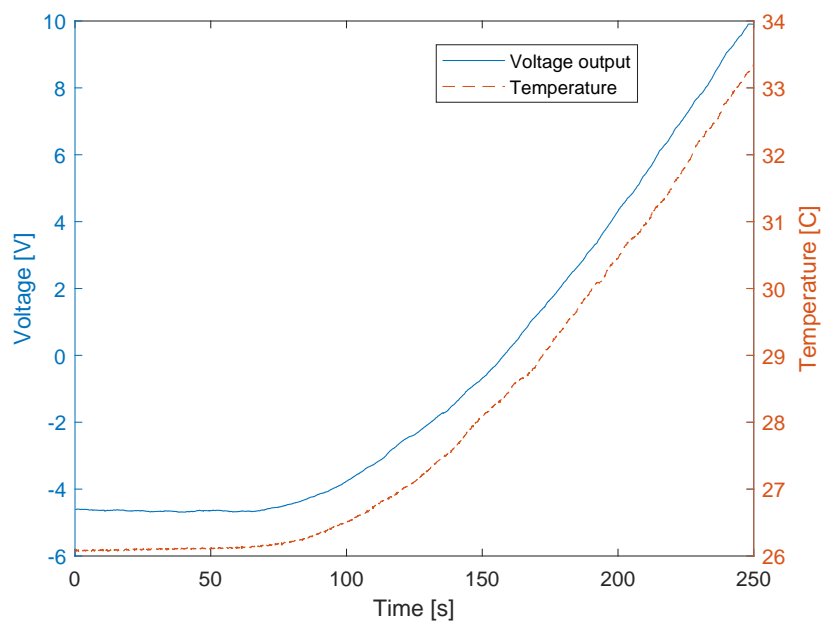

Fig. 6. Temperature and voltage output of the circuit.

\section{Mechanical Design Optimization}

Early prototypes of the sensor (Gen. 1) used a $3.9 \mathrm{~mm} \mathrm{x}$ $1.7 \mathrm{~mm}$ flap with a relatively simple hinge design with two 20 $\mu \mathrm{m}$ wide legs with height $H$ and an open area between them that led to some of the fluid to bypass the sensor.( See Figure 8 left). The present version utilizes a flap that is extended 


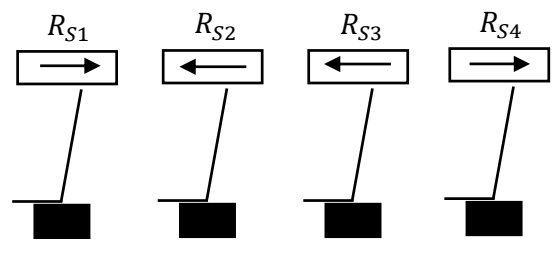

Fig. 7. Orientation of the MTJ sensors along the flow channel.

down to the substrate. This design reduces the bypass area and allows for longer, more flexible hinges.(See Figure 8 right) Having excessively long legs is also undesirable, as it leads to bending in the opposite direction due to the shift of the center of pressure. To determine the optimal value of $H$, finite element-based optimization procedure was performed using commercially available software (CFX, Ansys Inc.). The FEA model contained 329,420 tetrahedral elements with zero-slip boundary conditions applied on the side walls of the channel, while the nodes at the outlet were subjected to zero relative pressure. A preset mass flow rate was applied to the nodes corresponding to the channel inlet. The resulting hinge length vs deflection is shown in Figure 8. During the optimization process the hinge length $H$ was varied from 50 to $1000 \mu \mathrm{m}$ in the case of the first generation transducer and from 200 to $3500 \mu \mathrm{m}$ for the second generation. During the optimization process the gap $G$ was kept constant at $100 \mu \mathrm{m}$ and the hinge width, $W$ was kept at $20 \mu \mathrm{m}$. From the figure we see that the maximum achievable deformation with the first generation design was $32.8 \mu \mathrm{m}$ with a hinge length of $0.35 \mathrm{~mm}$ while the second generation allowed for $159 \mu \mathrm{m}$ of deflection with a $2 \mathrm{~mm}$ long hinge at $100 \mathrm{ml} / \mathrm{h}$. This change represents a 4.8 fold increase in sensitivity. The deflection mode shapes as a function of hinge length are shown in Figure 9.

This design $(H=2 \mathrm{~mm}, W=20 \mu \mathrm{m})$ proved to be challenging to manufacture and implement as the large aspect ratio of the legs caused it to be very fragile during the assembly process and even after successful assembly the pulling force of the permanent magnet caused buckling to occur in the legs, thus hindering the mobility of the transducer. The buckling of the legs caused the bottom of the transducer to latch against the bonding pad, limiting flexibility at low flow rates. At higher flow rates (above $300 \mathrm{ml} / \mathrm{h}$ ), the fluid pressure was large enough to release the top part of the transducer from the bonding pad, which resulted in a sudden voltage change in the signal output. In order to reduce the aspect ratio of the hinges while still retaining the reliability of the original design and improving on the sensitivity, the width of the hinges $W$ was increased from $20 \mu \mathrm{m}$ to $50 \mu \mathrm{m}$. The FEA optimization was also performed with the $50 \mu \mathrm{m}$ wide hinges and the maximum deflection was fount to be $55.9 \mu \mathrm{m}$ at $H=2 \mathrm{~mm}$ hinge height. While the overall sensitivity was reduced, this change still represents a 1.7 fold increment over the first generation design.

One concern regarding the introduction of multiple transducers in series is the increased pressure pressure drop across the sensor. Based on the FEA stated above, it has been shown that the pressure drop across the four transducers is 0.018 $\mathrm{mmHg}$, which represents a $0.7 \%$ increase over the nominal pressure drop of a typical $1.2 \mathrm{~m}$ long shunt tubing.

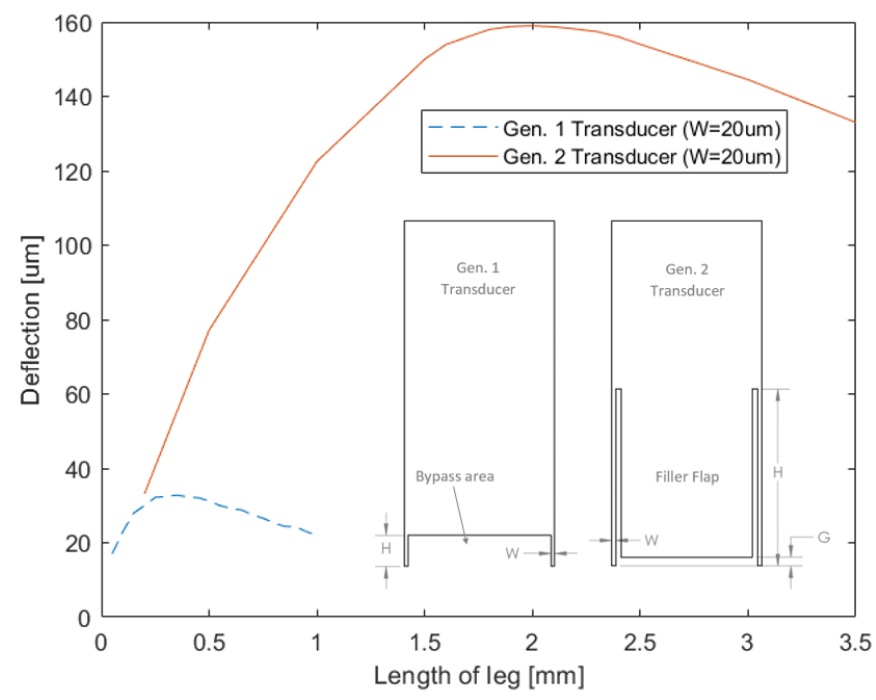

Fig. 8. Optimization of hinge length.

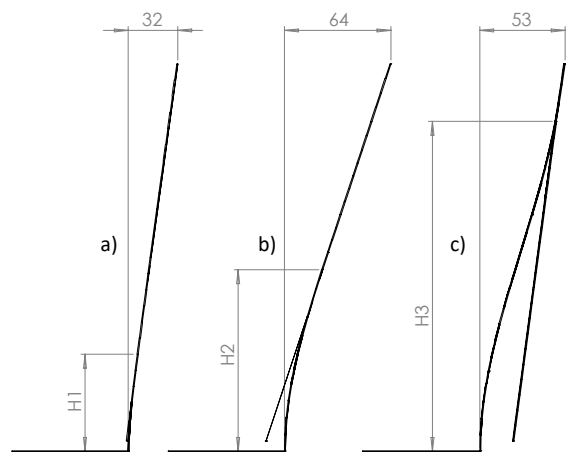

Fig. 9. Deflection values in $\mu \mathrm{m}$ and mode shapes at hinge lengths of a) $\mathrm{H} 1=0.5 \mathrm{~mm}, \mathrm{~b}) \mathrm{H} 2=2 \mathrm{~mm}, \mathrm{c}) \mathrm{H} 3=3.5 \mathrm{~mm}$ from FEA analysis for $100 \mathrm{ml} / \mathrm{h}$ (16x scale deformation).

\section{System integration and evaluation}

To test the flow transducers, a custom low power data acquisition board has been developed. The board contains the four MTJ sensors, Wheatstone bridge with digital tuning rheostats, an AD8426 Instrumentational amplifier for two stage signal amplification including a low pass filter, temperature sensor, $512 \mathrm{kB}$ of EEPROM memory and a 12 bit ADC connected to an Atmega 328P microcontroller. The circuit block diagram and the circuit board is shown in Figures 10 and 11. Low power consumption of the circuit is achieved by cycling between sleep and wake modes between sample points with a $3 \%$ duty cycle. The built in memory allows for 196608 data points to be collected over 24 hours at $2 \mathrm{~Hz}$ sample rate, although higher sample rates and larger memory modules are possible to implement. 
The glass substrate carrying the transducers has been placed in a $2 \mathrm{~mm} \times 4 \mathrm{~mm}$ milled acrylic channel with $0.81 \mathrm{~mm}$ ID silicone tube inlet and outlet leads. A syringe pump was used to generate flow rates between $1-50 \mathrm{ml} / \mathrm{h}$. The pump was alternating between flow and no flow regimes in order to acquire a calibration curve. Data was collected at $2 \mathrm{~Hz}$ sample rate and stored in the non-volatile memory on the data acquisition board. Higher sample rates are possible, at the expense of greater power consumption requiring larger batteries. Wireless data transfer using near-field communication (NFC) protocol has been proven, though for convenience during testing USB data transfer was used. In addition, 24 hour data samples were collected at constant flow rates to establish sensor drift values.

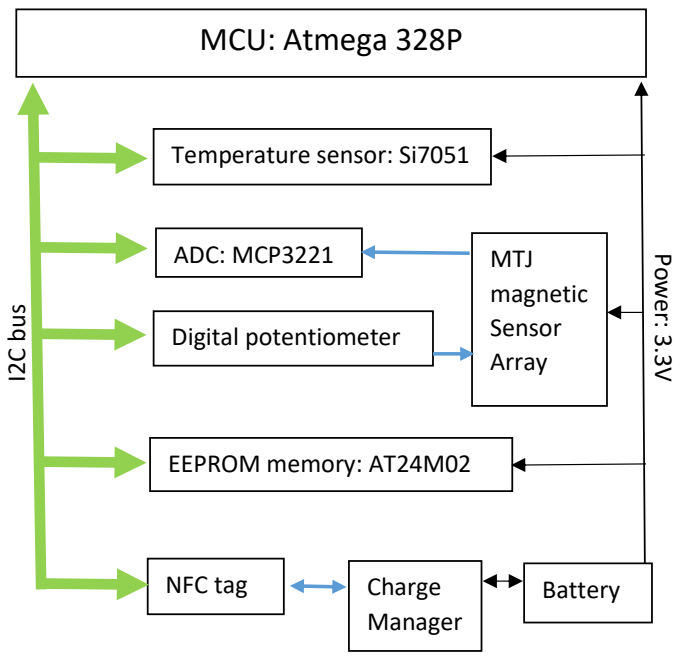

Fig. 10. Data acquisition circuit block diagram.

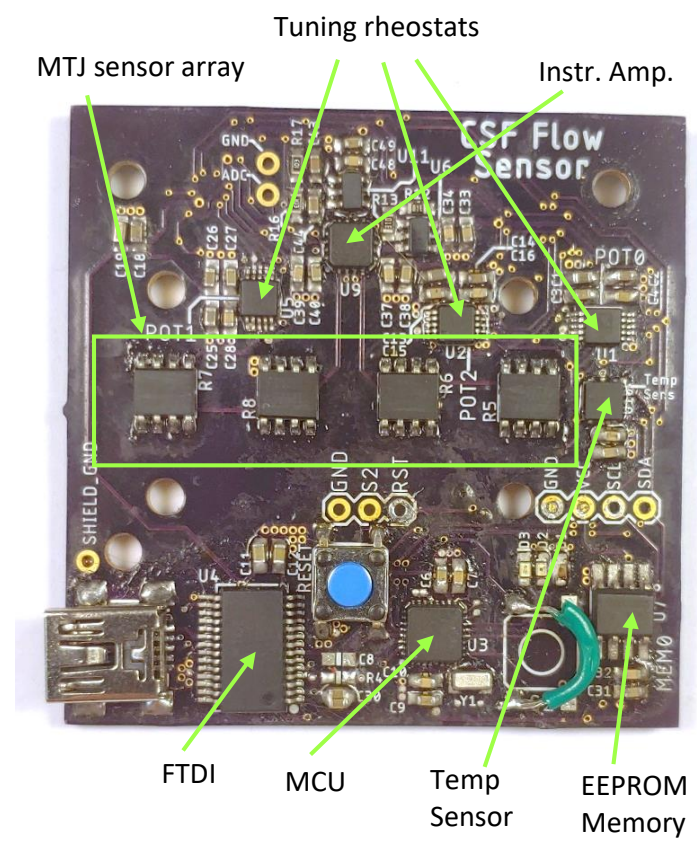

Fig. 11. Data acquisition circuit.
Raw data output is shown in Figure 12 at $20 \mathrm{ml} / \mathrm{h}$. Correlating the voltage levels at various flow rates a linear relationship can be observed. The slope of the curve represents the sensitivity of the transducer. As anticipated, combining the benefits redesigned transducer and the multi-sensor array the sensitivity has improved from $25 \mathrm{mV} / \mathrm{m} / \mathrm{h}$ to $272 \mathrm{mV} / \mathrm{ml} / \mathrm{h}$ without increasing the noise level. This corresponds to a 10.9 fold increment in signal to noise ratio compared to previous efforts. Figure 13 shows the circuit response at different flow rates. Repeated measurements showed an uncertainty of $4 \%$ RMS with respect to a linear curve and a resolution of 0.4 $\mathrm{ml} / \mathrm{h}$. The increased sensitivity allowed for a reduction of signal amplification from 3000 to 700, further reducing the temperature sensitivity of the device from $1.1 \mathrm{~V} /{ }^{\circ} \mathrm{C}$ to 0.3 $\mathrm{V} /{ }^{\circ} \mathrm{C}$.

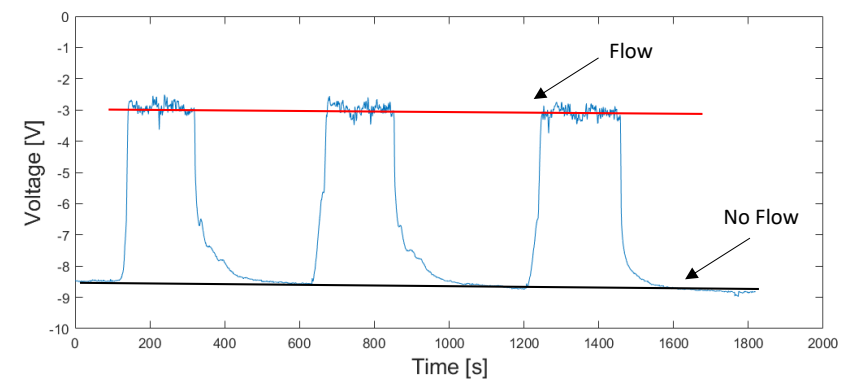

Fig. 12. Sensor output during flow and no-flow regimes at $20 \mathrm{ml} / \mathrm{h}$.

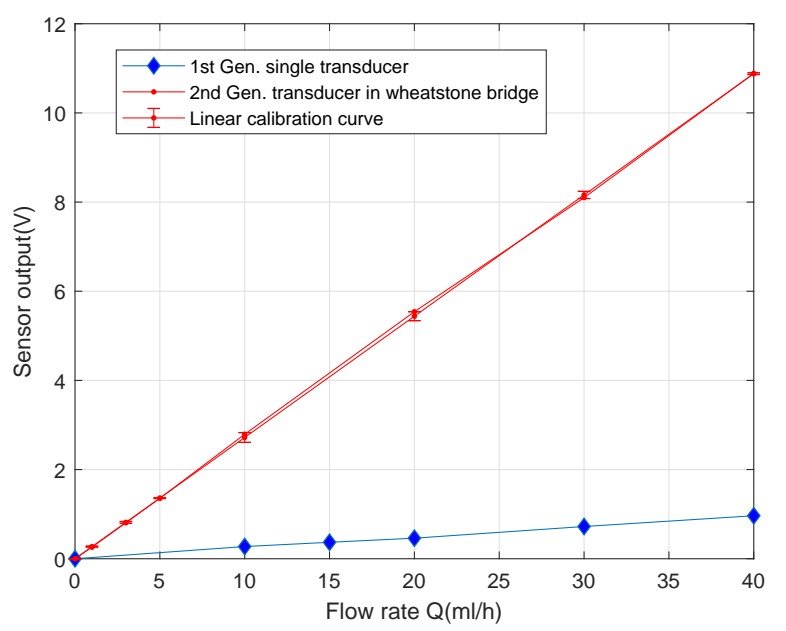

Fig. 13. Calibration curves comparing one sensor vs four sensors.

In order to quantify the amount of thermal drift over a 24-h period, the signal data was integrated to obtain volume fluctuation over a $24-\mathrm{h}$ period (under net zero flow). Figure 14 shows the results of the integration where we can see that the maximum measurement error during the day is $+2 \mathrm{ml}$.

In adults the upper limit of normal intracranial pressure is 15 $\mathrm{mmHg}$ and the lower limit of elevated pressure is $20 \mathrm{mmHg}$, therefore a sensitivity within $\pm 5 \mathrm{mmHg}$ [15] should allow to 
detect volumetric errors leading to clinically significant elevations in intracranial pressure. A typical patient has an average brain pressure-volume compliance of $0.68 \mathrm{ml} / \mathrm{mmHg}$ [16], and assuming that they require total CSF diversion due to complete CSF resorption obstruction, the device is required to detect intracranial pressure changes of $5 \mathrm{mmHg}$, which corresponds to the accumulation of $3.4 \mathrm{ml}$ of additional volume within the CSF space. Figure 14 shows the results of four separate 24-hour long zero-flow tests. The resulting volumetric error is $+0.5 /-1.5 \mathrm{ml}$ which stays below the critical value of 3.4 $\mathrm{mL}$, corresponding to $\pm 5 \mathrm{mmHg}$ pressure fluctuation. Thus, the proposed sensor has sufficient accuracy for taking flow measurements without clinically significant elevations of ICP.

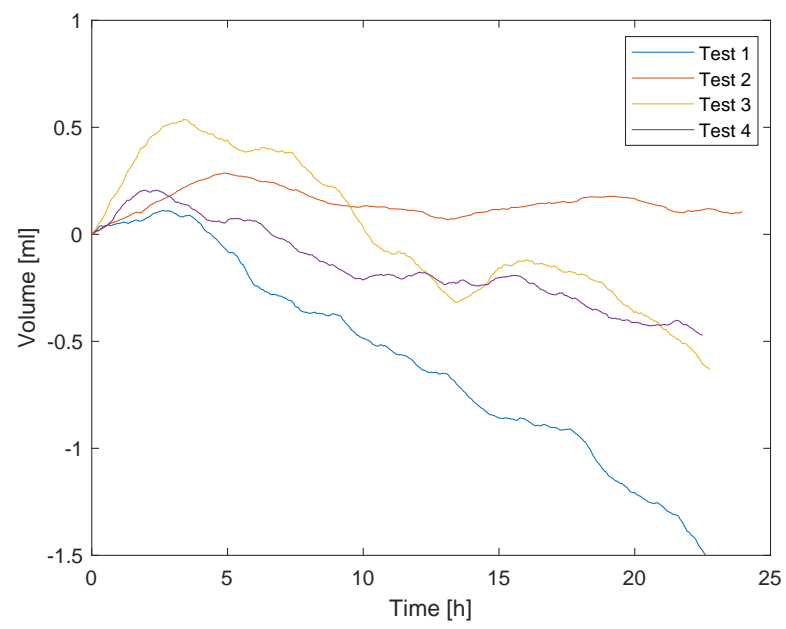

Fig. 14. Volume error during multiple 24-hour periods.

\section{E. Electromagnetic shielding}

Another issue presented in the previous analysis was the presence of the high frequency noise induced by electromagnetic fields. In order to decrease the amount of noise, an electromagnetic Faraday cage (shield) was placed around the sensors. The shield is made of a $0.25 \mathrm{~mm} \mathrm{Mu-metal} \mathrm{material,}$ which is able to reduce the amplitude of frequencies above 30 $\mathrm{Hz}$ according to the skin effect equation [17]. After placing the shield around the sensors the amplitude of the random noise picked up by the sensors has been reduced from $400 \mathrm{mV}$ to $100 \mathrm{mV}$. This improves the resolution of high frequency flow sensing from $1.5 \mathrm{ml} / \mathrm{h}$ to $0.4 \mathrm{ml} / \mathrm{h}$. The comparison between the signals with and without the shield is shown in Figure 15. In order to achieve the $1 \mathrm{ml} / \mathrm{h}$ resolution stated in the design specifications, the use of the shield is necessary.

\section{F. Corrosion Analysis}

Another concern of the thin nickel transducer design is its corrosion resistance and the health concerns about nickel ions entering the human body. The maximum allowable nickel intake per day for the human body is $128-137 \mu \mathrm{g}$ [18], the weight of one nickel transducer is $5.85 \mu \mathrm{g}$ which indicates that gradual corrosion of the transducer cannot cause harm
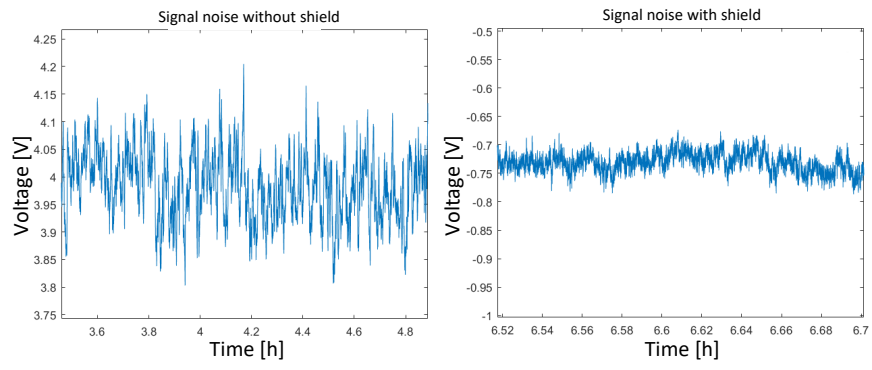

Fig. 15. Signal noise with and without electromagnetic shielding.

to the patient's health, however excessive corrosion could led to structural degradation of the transducer thus compromising its functionality. In order to determine the corrosion rate of the nickel transducers, a corrosion analysis was performed using cyclic voltammetry. The test was performed using the Gamry PCI4/300 potentiostat and an electrolytic cell. The cell consisted of a graphite counter electrode a cylindrical nickel working electrode with and without synthetic resin coating and $\mathrm{Ag} / \mathrm{Agcl}$ reference electrode. The resin coat was applied by dip coating method, resulting in a $70 \mu \mathrm{m}$ thick layer on the $0.47 \mathrm{~mm}$ diameter nickel electrode. The solution used in the cell was 1000 ppm $\mathrm{NaSO}_{4}$ with a pH level of 6.85 which is close to the $\mathrm{pH}$ level of CSF, thus providing a good representation of an implanted device. The test was repeated multiple times with and without a corrosion resistant coating on the nickel. The model we use for the corrosion rate calculations is based on Tafel equation [19] which is expanded for anodic-cathodic reactions resulting in a corrosion model based on Butler-Volmer equation Eq. (1)

$$
I=I_{\mathrm{corr}}\left(e^{\frac{2.303\left(E-E_{\mathrm{corr}}\right)}{\beta_{a}}}-e^{\frac{-2.303\left(E-E_{\mathrm{corr}}\right)}{\beta_{c}}}\right),
$$

where $I$ is the measured current from the cell, $I_{\text {corr }}$ is the corrosion current, $E$ is the electrode potential, $E_{\text {corr }}$ is the corrosion potential and $\beta_{a}$ and $\beta_{c}$ are the anodic and cathodic Tafel constants. The resulting I-V curve in logarithmic scale is shown in Figure 16. The corrosion current is than obtained by extrapolating the linear portions of the logarithmic curves in Figure 16 and determining the current at their intersection point for the anodic and cathodic regime and taking the average of the two. The corrosion rate is then calculated from Eq. (2), where $I_{\text {corr }}$ is the corrosion current, $K$ defines the units for the corrosion rate (in our case $K=3272 \mathrm{~A}-\mathrm{cm}$ year), $W_{e}$ is the equivalent weight $d$ is the density and $A$ is the sample area.

$$
C_{R}=\frac{I_{c o r r} \cdot K \cdot W_{e}}{d \cdot A}
$$

The tabulated results for the measured corrosion rates can be seen in Table I below and the raw output of the cyclic voltammety is in Figure 16. Data from the table indicate a lifespan for the uncoated and coated $10-\mu \mathrm{m}-$ thick transducer element is 15 and 55 years respectively. In the event of material failure due to corrosion, the dislodged transducer would not enter the patients peritoneal cavity as the dimensions of the 
transducer exceed the diameter of the shunt tubing. This breakage would immediately be detected by the MTJ sensors.

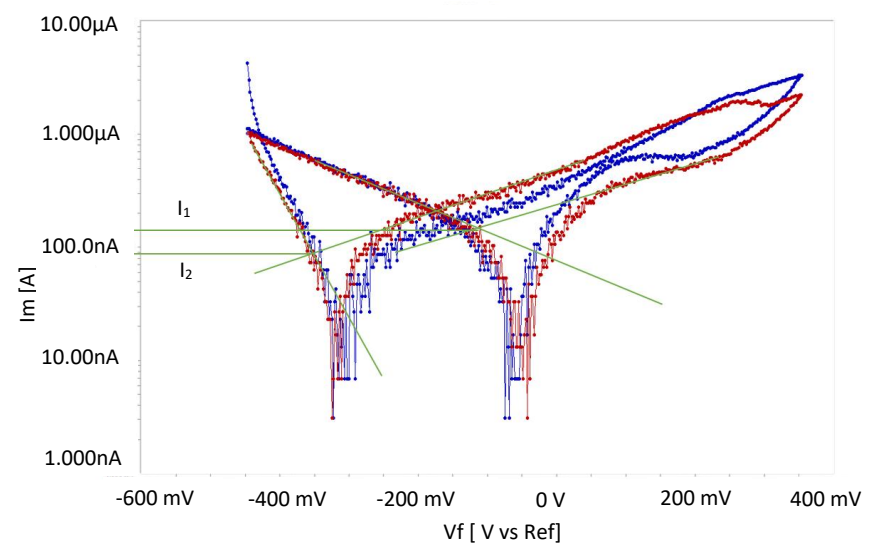

Fig. 16. Corrosion analysis output.

TABLE I

CORROSION ANALYSIS RESULTS.

\begin{tabular}{|l|l|l|}
\hline & $I_{\text {corr }}$ & $C_{R}$ \\
\hline Test 1 (Uncoated) & $22 \mathrm{nA}$ & $0.24 \mathrm{um} / \mathrm{year}$ \\
\hline Test 2 (Uncoated) & $30 \mathrm{nA}$ & $0.32 \mathrm{um} / \mathrm{year}$ \\
\hline Test 3 (Coated) & $12 \mathrm{nA}$ & $0.13 \mathrm{um} / \mathrm{year}$ \\
\hline Test 4 (Coated) & $9 \mathrm{nA}$ & $0.09 \mathrm{um} / \mathrm{year}$ \\
\hline
\end{tabular}

\section{G. Dynamic Flow Testing}

Given the anticipated pulsatile nature of the CSF flow, the sensor will likely be exposed to variable flows with frequency near that of the heart. Therefore analysis of its performance under variable flow conditions was undertaken. Transient response of the sensor has been investigated by subjecting it to a flow impulse produced by dropping a small $(15 \mathrm{~g})$ proof mass onto a syringe plunger. In order to determine the bandwidth of the sensor, a second order model was fitted to the data using system identification approach. Figure 17 shows the impulse response of the sensor and the response of the model. The Bode analysis of the resulting system revealed a $3 \mathrm{~Hz}$ bandwidth, which corresponds to the cutoff frequency of the low-pass filter in the data acquisition board. This means the mechanical design of the transducers is not the limiting factor in the system bandwidth (Figure 17).

\section{Discussion AND CONClusions}

In this paper a self-contained high-frequency highsensitivity implantable flow sensor was presented based on the principles of magnetic field sensing. Optimization of the geometry of previously proposed flow sensor demonstrated increased sensitivity and signal to noise ratio. Low frequency drift was shown to be due to thermal fluctuations impacting the resistors in the bridge and the operational amplifiers. When four MTJ sensors with matched TCR coefficients are used in the Wheatstone bridge, the temperature induced signal drift is reduced to $2 \mathrm{ml}$ per 24-hour period.

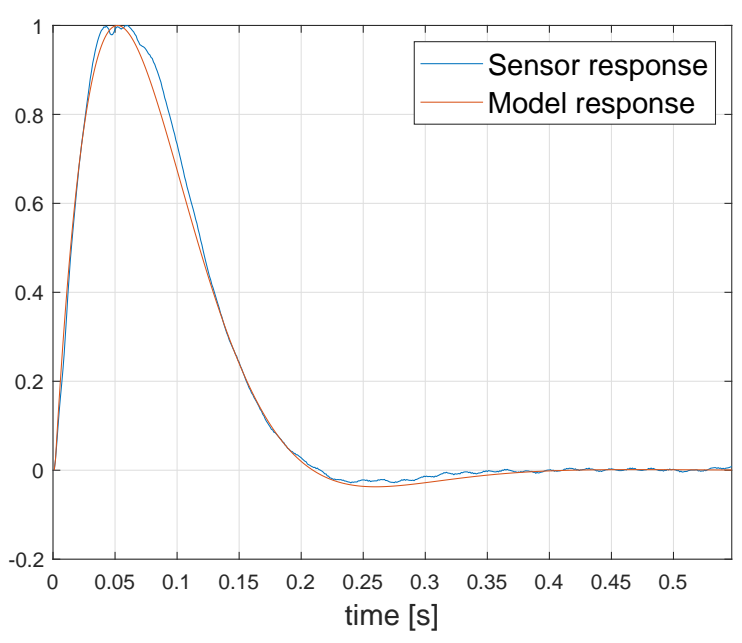

Fig. 17. Transient impulse response of flow sensor.

The present study showed that it is possible to increase the sensitivity of the MTJ-based flow sensor by changing the architecture of the ferromagnetic transducer. Experimental validation of the design was also presented using a custom data acquisition circuit board, allowing for user-adjustable data collection rates, wireless data transfer, low noise and low drift. Repeated flow measurements showed an uncertainty of $4 \%$ RMS and a resolution of $0.4 \mathrm{ml} / \mathrm{h}$. It was also shown that the suspected thermal sensitivity of the sensor is present in both the MTJ sensors as well as in the amplification circuit. Through the use of four MTJ magnetic sensors with equal TCR values connected in the Wheatstone bridge, it is possible to reduce the low frequency temperature related signal drift down to $2 \mathrm{ml}$ per 24-hour period. These values are below the allowable volume change for the ventricles of an adult brain. The sensitivity of the sensor device has also been improved by the introduction of a full-bridge sensing circuit with four magnetic transducers, increasing the sensitivity 10.9 fold. Through the introduction of electromagnetic shielding an improvement in the signal to noise ratio by a factor of 4 was achieved. A corrosion analysis has been performed indicating a possible 15 to 55 years of life span of the device. The dynamic response of the sensor appears adequate for the intended application.

\section{ACKNOWLEDGMENT}

The authors acknowledge the support for this research by a Grant\# 1705761 from the National Science Foundation. None of the authors have any financial/competing interests in connection with this manuscript and the findings reported in it. There were no human subjects involved in this study.

\section{REFERENCES}

[1] Y. Wu, N. L. Green, M. R. Wrensch, S. Zhao, and N. Gupta, "Ventriculoperitoneal shunt complications in california: 1990 to 2000," Neurosurgery, vol. 61, no. 3, pp. 557-563, 2007.

[2] H. J. Garton, J. R. Kestle, and J. M. Drake, "Predicting shunt failure on the basis of clinical symptoms and signs in children," Journal of neurosurgery, vol. 94, no. 2, pp. 202-210, 2001. 
[3] J. M. Drake, J. R. Kestle, R. Milner, G. Cinalli, F. Boop, J. Piatt Jr, S. Haines, S. J. Schiff, D. D. Cochrane, P. Steinbok et al., "Randomized trial of cerebrospinal fluid shunt valve design in pediatric hydrocephalus," Neurosurgery, vol. 43, no. 2, pp. 294-303, 1998.

[4] J. Kestle, J. Drake, R. Milner, C. Sainte-Rose, G. Cinalli, F. Boop, J. Piatt, S. Haines, S. Schiff, D. Cochrane et al., "Long-term followup data from the shunt design trial," Pediatric neurosurgery, vol. 33 , no. 5 , pp. 230-236, 2001

[5] L. Acakpo-Satchivi, C. N. Shannon, R. S. Tubbs, J. C. Wellons III, J. P. Blount, B. J. Iskandar, and W. J. Oakes, "Death in shunted hydrocephalic children: a follow-up study," Child's Nervous System, vol. 24, no. 2, pp. 197-201, 2008.

[6] R. V. Patwardhan and A. Nanda, "Implanted ventricular shunts in the united states: the billion-dollar-a-year cost of hydrocephalus treatment," Neurosurgery, vol. 56, no. 1, pp. 139-145, 2005.

[7] J. R. Atkinson, D. B. Shurtleff, and E. L. Foltz, "Radio telemetry for the measurement of intracranial pressure." Journal of neurosurgery, vol. 27, no. 5 , p. 428,1967

[8] V. Macellari, "Batteryless on-demand-sampling active radiosonde for intracranial pressure measurement," Medical and Biological Engineering and Computing, vol. 19, no. 6, pp. 686-694, 1981.

[9] T. Bork, A. Hogg, M. Lempen, D. Müller, D. Joss, T. Bardyn, P. Büchler H. Keppner, S. Braun, Y. Tardy, and J. Burger, "Development and invitro characterization of an implantable flow sensing transducer for hydrocephalus," Biomedical Microdevices, vol. 12, pp. 607-618, 2010.

[10] C. Qin, A. H. Olivencia-Yurvati, A. G. Williams Jr, D. Eskildsen, R. T Mallet, and P. K. Dasgupta, "Inline flow sensor for ventriculoperitoneal shunts: Experimental evaluation in swine," Medical engineering \& physics, vol. 67, pp. 66-72, 2019.

[11] T. Hudson, A. Baldwin, E. Christian, J. G. McComb, and E. Meng, "Testing a multi-sensor system for hydrocephalus monitoring in external ventricular drains," in 2019 20th International Conference on SolidState Sensors, Actuators and Microsystems \& Eurosensors XXXIII (TRANSDUCERS \& EUROSENSORS XXXIII). IEEE, 2019, pp. 310313.

[12] R. Raj, S. Lakshmanan, D. Apigo, A. Kanwal, S. Liu, T. Russell, and R. C. Farrow, "Demonstration that a new flow sensor can operate in the clinical range for cerebrospinal fluid flow," Sensors and actuators, vol. 234, pp. 223-231, 2015.

[13] E. T. Enikov, G. . 'Edes, J. Skoch, and R. Anton, "Application of gmr sensors to liquid flow sensing," Journal of Microelectromechanical Systems, vol. 24, no. 4, pp. 914-921, 2015.

[14] R. Hftberger, S. A. Mader, and M. Reindl, Cerebrospinal Fluid in Clinical Neurology, 012015.

[15] J. Miller and J. Garibi, "Intracranial volume/pressure relationships during continuous monitoring of ventricular fluid pressure," in Intracranial pressure. Springer, 1972, pp. 270-274.

[16] G. Portella, M. Cormio, G. Citerio, C. Contant, K. Kiening, P. Enblad, and I. Piper, "Continuous cerebral compliance monitoring in severe head injury: its relationship with intracranial pressure and cerebral perfusion pressure," Acta neurochirurgica, vol. 147, no. 7, pp. 707-713, 2005.

[17] E. C. Jordan, Electromagnetic waves and radiating systems. PrenticeHall, 1968.

[18] A. for Toxic Substances and D. Registry, Toxicological Profile for Nickel. U.S.Public Health Service, 1993.

[19] P. H. Reiger, Electrochemistry. Springier, 1994.

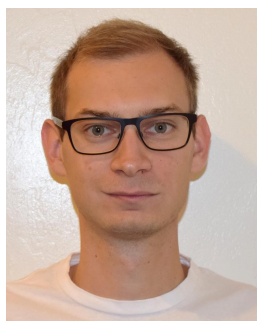

Gergó Édes received his M.S. degree in mechanical engineering from the Slovak University of Technology, Bratislava, Slovakia in 2014, and completed a one year research program at the University of Arizona in 2013 involving the development of MTJ magnetic sensor based implantable flow sensors for hydrocephalus patients. Since January 2015 he has been working towards a $\mathrm{Ph} . \mathrm{D}$. degree from Mechanical Engineering at the University of Arizona.

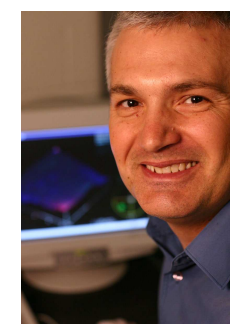

Eniko T. Enikov is a professor of mechanical and aerospace engineering at the University of Arizona. He received his MS degree from Technical University of Budapest in 1993 and $\mathrm{PhD}$ degree from University of Illinois at Chicago in 1998. His research is focused on the design and fabrication of micro-electromechanical systems (MEMS) with application to eye tonometery, glaucoma shunts, and various drug delivery devices. He is also interested in non-linear dynamic phenomena at the micro- and nanoscale. Dr. Enikov is a member of the professional societies of ASME and ASEE.

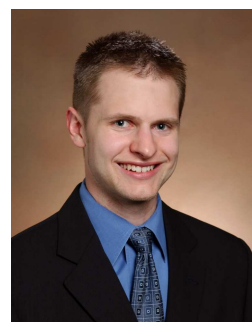

Jesse Skoch is completing his neurosurgical residency at the University of Arizona and plans to begin a pediatric neurosurgery fellowship in 2015 . Prior to his medical training he performed research at Harvard / Mass General Hospital primarily working with mouse models of Alzheimers disease and in using multiphoton microscopy to assist with in vivo detection of Alzheimer plaque pathology. His current research interests focus on integrating optical imaging techniques and engineering solutions into clinical practice to improve the diagnosis and treatment of neurological diseases such as brain aneurysms and hydrocephalus.

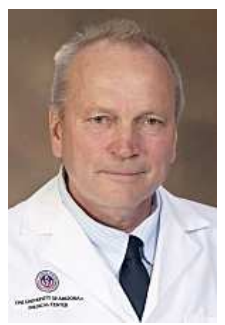

Rein Anton is an assistant professor of surgery in the Department of Surgery, College of Medicine, University of Arizona. His present research interests involve spine and spinal cord trauma and especially complex surgeries to determine the most appropriate surgical approaches for these conditions. 